\title{
Peripheral Vascular Dysfunction in Chronic Kidney Disease
}

\author{
Christopher R. Martens and David G. Edwards \\ Department of Kinesiology \& Applied Physiology, University of Delaware, 541 South College Avenue, Newark, DE 19716, USA
}

Correspondence should be addressed to David G. Edwards, dge@udel.edu

Received 24 December 2010; Accepted 15 March 2011

Academic Editor: Abarmard Maziar Zafari

Copyright ( $) 2011$ C. R. Martens and D. G. Edwards. This is an open access article distributed under the Creative Commons Attribution License, which permits unrestricted use, distribution, and reproduction in any medium, provided the original work is properly cited.

\begin{abstract}
There is an increased prevalence of cardiovascular disease- (CVD-) related mortality in patients with chronic kidney disease (CKD). Endothelial dysfunction is a primary event in the development of atherosclerosis and hypertension and likely contributes to the elevated cardiovascular risk in CKD. Endothelial dysfunction has been shown to occur in the peripheral vasculature of patients with both severe and moderate CKD. Mechanisms include oxidative stress, L-arginine deficiency, and elevated plasma levels of ADMA. Interventions designed to restore vascular function in patients with CKD have shown mixed results. Evidence from cell culture studies suggest that the accumulation of uremic toxins inhibits L-arginine transport and reduces nitric oxide production. The results of these studies suggest that endothelial dysfunction may become less reversible with advancing kidney disease. The purpose of this paper is to present the current literature pertaining to potential mechanisms of peripheral vascular dysfunction in chronic kidney disease and to identify possible targets for treatment.
\end{abstract}

\section{Introduction}

Chronic kidney disease (CKD) is a major public health concern affecting nearly 20 million people in the United States alone [1]. Patients with CKD are at greater risk of cardiovascular disease- (CVD-) related morbidity and mortality than individuals without CKD with similar cardiovascular risk factors and tend to die before reaching end-stage renal disease (ESRD) [2-5]. Treatments aimed solely at reducing traditional cardiovascular risk factors do not improve cardiovascular function in patients with latestage CKD [6]. Therefore, traditional CVD risk factors alone cannot explain the high incidence of CVD in CKD.

Endothelial dysfunction is a precursor to the development of atherosclerosis $[7,8]$ and has been shown to be associated with increased cardiovascular risk in patients with left ventricular dysfunction and congestive heart failure [911]. The combination of traditional risk factors in CKD is not enough to explain the high incidence of CVD and endothelial dysfunction has been suggested to play a role in the increased CV risk in CKD $[12,13]$. In support of this, a longitudinal study of patients with end-stage renal disease (ESRD) found all cause mortality to be independently associated with impaired endothelial function [14].

The majority of studies of endothelial function in renal disease have focused on ESRD and patients receiving dialysis; however, little is known about endothelial function in earlier stages of CKD. Guidelines from the Kidney Disease Outcomes Quality Initiative (K/DOQI) place patients with CKD into one of five stages based on glomerular filtration rate (GFR) and are presented in Table $1[1,15]$. The risk of developing CVD can be predicted from GFR and increases as GFR declines [3]. These guidelines make it easier to delineate differences in vascular function in CKD patients with low to moderate renal deficiency; however to date those data are limited. With the majority of patients dying of cardiovascular disease before needing dialysis, there is an urgent need for investigations into vascular function in earlier stages of CKD. A great deal of literature has described the role of vascular calcification and aortic stiffness in promoting atherosclerosis in CKD and have been reviewed elsewhere [16]. The purpose of this paper is to present a summary of the available research regarding mechanisms of peripheral vascular dysfunction throughout the progression of chronic kidney disease. 
TABLE 1: Stages of CKD.

\begin{tabular}{llc}
\hline Stage & Description & $\begin{array}{c}\text { GFR, } \mathrm{mL} \cdot \mathrm{min}^{-1} \text { per } \\
1.73 \mathrm{~m}^{2}\end{array}$ \\
\hline 1 & $\begin{array}{l}\text { Kidney damage with } \\
\text { normal or increased GFR }\end{array}$ & $\geq 90$ \\
& $\begin{array}{l}\text { Kidney damage with mildly } \\
\text { decreased GFR }\end{array}$ & $60-89$ \\
3 & Moderately decreased GFR & $30-59$ \\
4 & Severely decreased GFR & $15-29$ \\
5 & Kidney failure & $<15$ or dialysis \\
\hline
\end{tabular}

Adapted from National Kidney Foundation K/DOQI clinical practice guidelines for chronic kidney disease [15].

\section{Vascular Function in CKD}

The vascular endothelium consists of a single layer of cells lining the interior lumen of blood vessels. Aside from functioning as a barrier between the intravascular space and the interstitum, the vascular endothelium works as an important regulator of vascular homeostasis. Perhaps one of the most important functions of the endothelium is the synthesis and release of the vasodilator nitric oxide [17]. Nitric oxide is produced from the amino acid substrate Larginine by the enzyme endothelial nitric oxide synthase (eNOS) [18]. Activation of eNOS requires the binding of the cofactor tetrahydrobiopterin $\left(\mathrm{BH}_{4}\right)$ as well as the presence of $\mathrm{Ca}^{2+} /$ Calmodulin for the synthesis of NO [19]. NO diffuses through the basal membrane of the endothelial cell into the smooth muscle where it activates the conversion of GTP to cyclic GMP by soluble guanylyl cyclase (sGC) signaling smooth muscle relaxation and vasodilation [20]. This pathway occurs in response to mechanical shear stress or the binding of bradykinin or acetylcholine to their receptors on endothelial cells and is vital for proper control and maintenance of blood pressure. Additionally, nitric oxide plays an important role by regulating vascular permeability, leukocyte adhesion [21], and smooth muscle proliferation [22], all of which play an important role in maintaining cardiovascular health [23]. Endothelial dysfunction is characterized by a reduced synthesis or bioavailability of NO and is a primary event in the development of atherosclerosis [24]. In vivo assessment of conduit artery and microvascular function through flow-mediated dilation (FMD) and venous occlusion plethysmography (VOP) have been used to assess vascular function in CKD (Table 2) and serve as measures of endothelial function.

Endothelial dysfunction is present in CKD and may explain the increased cardiovascular risk in these patients. Urinary nitrate and nitrite (NOx) excretion, an index of total body NO production, is lower in patients with ESRD and likely contributes to the hypertension observed in these patients [36]. Endothelial dysfunction has been shown to occur in vitro in human microvessels obtained via subcutaneous fat biopsies from patients receiving dialysis or renal replacement surgery [37] as well as in vivo in the forearm microvasculature of predialysis CKD patients using venous occlusion plethysmography [28]. Further, endothelial dysfunction has been shown to occur in conduit vessels upstream of the microvasculature [27,34]. Thus, endothelial dysfunction appears to be characteristic of CKD.

The confounding effects of other risk factors in CKD make it difficult to differentiate whether the source of endothelial dysfunction is due to renal impairment or the combination of multiple risk factors. Studies using children with CKD have assessed endothelial function before atherosclerosis and other associated risk factors were believed to occur [38-40]. Endothelial-dependent dilation was shown to be impaired in children with chronic renal failure using flow-mediated dilation (FMD) suggesting that kidney disease alone leads to the development of endothelial dysfunction [38]. Additionally, nitric oxide plays an important role in attenuating renal impairment and may slow the progression of CKD in patients with mild renal impairment. Reduced NO bioavailability has been shown to contribute to glomerular hypertension and accelerate renal damage leading to more rapid progression of CKD [41]. While the presence of endothelial dysfunction is well documented in CKD, the mechanisms have not yet been fully explained.

\section{Potential Mechanisms of Endothelial Dysfunction in CKD}

3.1. Oxidative Stress. One of the most widely studied contributors to endothelial dysfunction is oxidative stress [42]. Oxidative stress is defined by a disruption in the balance between free radical production and removal by endogenous antioxidants [43]. Oxidative stress impairs NO signaling pathways in endothelial cells and contributes to endothelial dysfunction [43]. Oxidative stress is present in patients with moderate to severe CKD $[32,44]$ as well as patients with ESRD or receiving hemodialysis [32, 45]. Endothelial dysfunction worsens with progressive loss of renal function and is associated with a variety of markers of oxidative stress including lipid hydroperoxides, oxidized glutathione, protein carbonyls and F2-isoprostanes [29, 31, 44, 45] as well as reduced antioxidant capacity $[46,47]$.

Markers of oxidative stress are inversely correlated with endothelial function in patients with CKD. In one study, brachial-artery flow-mediated dilation was decreased in hemodialysis patients and correlated to increased levels of TBARS, a marker of lipid peroxidation [33]. Interestingly, these patients lacked overt symptoms of cardiovascular disease suggesting that oxidative stress may contribute to the early progression of endothelial dysfunction in CKD. Endothelial function and GFR have also been shown to be associated with increased levels of advanced glycation end products (AGEs) in different stages of predialysis, nondiabetic CKD, likely through increased abundance of AGE receptors (RAGE) on the surface of endothelial cells [48]. AGEs have been shown to increase inflammation and oxidative stress [48] and can also inhibit eNOS activity directly as well as reduce substrate bioavailability by reacting with L-arginine resulting in endothelial dysfunction [49].

The mechanisms behind the contribution of oxidative stress in endothelial dysfunction in CKD appear to occur primarily through eNOS and NO dependent pathways 
TABLE 2: Summary of studies measuring in vivo endothelial function in adult patients with CKD.

\begin{tabular}{|c|c|c|c|}
\hline Study & $\begin{array}{l}\text { Reported degree of } \\
\text { CKD }\end{array}$ & Method & Finding \\
\hline Yilmaz et al. [25] & Stage 1 & FMD & Improved following 12-week treatment with ramipril \\
\hline Yilmaz et al. [26] & Stage $1-4$ & FMD & Improved following 3-month treatment with ramipril or valsartan \\
\hline Nanayakkara et al. [27] & $\mathrm{CCr}=38 \pm 15$ & FMD & $\begin{array}{l}\text { Improvement following } 18 \text {-month stepwise treatment with pravastatin, } \\
\text { vitamin E, and homocysteinie lowering therapy }\end{array}$ \\
\hline Annuk et al. [28] & $\mathrm{CCr}=29.4 \pm 24.0$ & VOP & Impaired and related to degree of renal impairment \\
\hline Annuk et al. [29] & $\mathrm{CCr}=25.1 \pm 16.2$ & VOP & Impairments correlated to markers of oxidative stress \\
\hline Annuk et al. [30] & Serum $\mathrm{Cr}=287 \pm 143$ & VOP & Improved by acute COX inhibition or L-arginine treatment \\
\hline Annuk et al. [31] & Stage $3-5$ & VOP & Negatively correlated to levels of lipid hydroperoxides (LOOH) \\
\hline Ghiadoni et al. [32] & $\begin{array}{l}\text { Stage } 3-5 \text {, } \\
\text { hemodialysis }\end{array}$ & FMD & $\begin{array}{l}\text { Acute vitamin C infusion restored impaired function in hemodialysis } \\
\text { but not in CKD }\end{array}$ \\
\hline Costa-Hong et al. [33] & ESRD & FMD & $\begin{array}{l}\text { Plasma TBARS levels associated with impaired endothelial-dependent } \\
\text { dilation in patients without symptoms of CVD }\end{array}$ \\
\hline Cross et al. [34] & $\begin{array}{l}\text { Predialysis } \\
\text { hemodialysis }\end{array}$ & VOP, FMD & $\begin{array}{l}\text { Acute infusion of vitamin } \mathrm{C} \text { improves endothelium-dependent dilation } \\
\text { in forearm resistance vasculature but not in brachial artery }\end{array}$ \\
\hline Cross et al. [35] & $\begin{array}{l}\text { Predialysis, } \\
\text { hemodialysis }\end{array}$ & VOP, FMD & $\begin{array}{l}\text { Local or systemic L-arginine infusion did not improve resistance or } \\
\text { endothelial-dependent dilation }\end{array}$ \\
\hline
\end{tabular}

FMD = flow-mediated dilation; VOP = venous occlusion plethysmography; $\mathrm{CCr}=$ creatinine clearance $\left(\mathrm{mL} / \mathrm{min} / 1.73 \mathrm{~m}^{2}\right) ; \mathrm{Serum} \mathrm{Cr}=$ serum creatinine $(\mu \mathrm{mol} / \mathrm{L})$.

(Figure 1). Cross et al. [34] observed a reduction in acetylcholine-induced forearm blood flow (venous occlusion plethysmography) in predialysis patients with ESRD (GFR $<20 \mathrm{mLmin})$. This impairment was ameliorated when the antioxidant vitamin $\mathrm{C}$ was infused through the brachial artery suggesting that the source of endothelial dysfunction was oxidative stress. This improvement was abolished when vitamin $\mathrm{C}$ was infused with the NOS inhibitor L-NMMA suggesting that the effect of oxidative stress on forearm blood flow is NO dependent. Interestingly, vitamin C did not cause an improvement in endothelial function in larger upstream conduit vessels as assessed by brachial and radial artery flowmediated dilation in both predialysis and dialysis patients with severe renal failure [34]. This finding suggests that some other mechanism contributes to endothelial dysfunction in late-stage kidney disease.

Although oxidative stress has been shown to be more severe in hemodialysis patients than in patients with stage 3-5 CKD [32], studies have shown oxidative stress to contribute to endothelial dysfunction earlier in CKD. Perfused mesenteric arteries from rats that underwent renal mass reduction have impaired vasodilation in response to acetylcholine within 3-10 days after surgery [50]. This impairment was restored by incubation with the antioxidant enzyme superoxide dismutase (SOD) [50] suggesting that oxidative stress also plays a role in endothelial dysfunction early in the progression of CKD. In human patients with moderate renal impairment, the forearm blood flow response to an arterial infusion of methacholine was attenuated [29]. These same patients also demonstrated increased levels of lipid hydroperoxides ( $\mathrm{LOOH}$ ) and an increased ratio of oxidized to reduced glutathione (GSSG/GSH). These markers of oxidative stress were correlated to both serum creatinine and forearm blood flow suggesting that oxidative stress

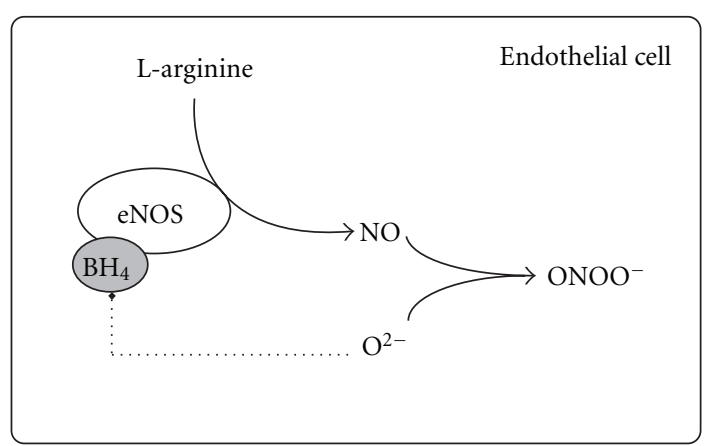

Figure 1: Simplified schematic depicting mechanisms by which reactive oxygen species can reduce nitric oxide $(\mathrm{NO})$ availability. $\mathrm{NO}$ is synthesized from eNOS and can combine with excess superoxide $\left(\mathrm{O}^{2-}\right)$ from vascular oxidases to form peroxynitrite $\left(\mathrm{ONOO}^{-}\right)$limiting $\mathrm{NO}$ availability. Superoxide can also oxidize the eNOS cofactor tetrahydrobiopterin $\left(\mathrm{BH}_{4}\right)$, uncoupling eNOS and reducing NO synthesis.

contributes to endothelial dysfunction in humans with moderate CKD.

Supplementation with antioxidants has been shown to reduce cardiovascular events in CKD and hemodialysis [51]. In the Secondary Prevention with Antioxidants of Cardiovascular Disease in Endstage Renal Disease (SPACE) trial, hemodialysis patients receiving $800 \mathrm{IU} /$ day of vitamin $\mathrm{E}$ had significantly fewer primary cardiovascular end-points than placebo-treated controls [52]. The benefits of antioxidant therapy have also been studied in earlier stages of CKD and have been shown to improve endothelial function. The Antioxidant Therapy in Chronic Renal Insufficiency (ATIC) 
study demonstrated that antioxidant treatment in patients with mild to moderate renal impairment was effective at improving brachial artery flow-mediated dilation [27]. Patients underwent three stages of drug treatment consisting of treatment with pravastatin, vitamin E, and homocysteinelowering therapy introduced consecutively at 6 month intervals. Additionally, this improvement in endothelial function occurred despite a progressive loss in renal function. Antioxidant treatment has not been effective at reducing cardiovascular risk in other diseases [53]; however, these studies suggest that the use of antioxidants in CKD may be an exception.

Another strategy for combating oxidative stress mediated endothelial dysfunction may be bolstering endogenous antioxidant defense mechanisms. Endogenous antioxidant enzymes play an important role in preventing oxidative stress and are impaired in CKD. Plasma and erythrocyte levels of glutathione peroxidase (GSH-px), superoxide dismutase (SOD), and catalase were shown to be significantly lower in predialysis patients with ESRD compared to healthy control subjects [46]. Endogenous antioxidants have also been shown to be lower in earlier stages of CKD with levels of erythrocytic GSH-px, and SOD impaired as early as stage 1 (GFR $<90 \mathrm{~mL} / \mathrm{min})$ [45]. Treatments aimed at improving the endogenous antioxidant defense systems may be more effective than antioxidant therapy. Chronic exercise training was shown to reduce levels of ROS and improve endothelial-dependent relaxation in 5/6 nephrectomized rats [54]. Exercise has been shown to improve endothelialdependent dilation in aging [55] and may help prevent the progression of endothelial dysfunction in CKD; however, further study is needed.

The role of oxidative stress on vascular function in CKD is well documented however more research is needed to uncover the sources and specific targets of free radicals. The production of the powerful radical superoxide from NADPH oxidases is a likely contributor oxidative stress in the endothelium [43] and can combine with $\mathrm{NO}$ to form reactive nitrogen species such as peroxynitrite $\left(\mathrm{ONOO}^{-}\right)$ (Figure 1). Peroxinitrite formation reduces the bioavailibilty of NO and contributes to nitrositive cellular damage. Vaziri et al. [56] measured increased nitrotyrosine abundance, a marker of nitrositive protein modification, in aorta, heart, liver, and plasma of 5/6 nephrectomized rats. Nitrotyrosine levels were decreased to normal levels in rats treated with the antioxidant vitamin E. Angiotensin II is elevated in CKD and has been shown to activate NADPH oxidases [57]. Few studies have examined the role of inhibition of the reninangiotensin system on endothelial-dependent dilation in CKD. One study observed improved FMD following shortterm ACE-inhibition in patients with stage 1 diabetic CKD [25]. Additional free radical production may occur from xanthine oxidase; however, the role of this enzyme in humans with CKD is not well known. Treatment with the xanthine oxidase inhibitor allopurinol slowed the progression of CKD, and reduced cardiovascular risk in patients with CKD (eGFR $<60 \mathrm{~mL} / \mathrm{min}$ ) [58]; however, it is unclear if these beneficial effects were due to decreased oxidative stress or reduced serum uric acid levels.
Oxidation of the essential eNOS cofactor tetrahydrobiopterin $\left(\mathrm{BH}_{4}\right)$ has been shown to cause uncoupling of eNOS (Figure 1). Once uncoupled, eNOS itself becomes a source of superoxide contributing to further oxidative damage $[59,60]$. Chronic treatment with $\mathrm{BH}_{4}$ has been shown to lower systolic blood pressure and reduce proteinuria in 5/6 nephrectomized rats [61]. In another study, endothelial-dependent relaxation of aortic rings from 5/6 nephrectomized rats was restored in isolated vessels treated with $\mathrm{BH}_{4}$ [62]. These vessels were also associated with increased superoxide production which was ameliorated by treatment with L-NAME suggesting that the source of oxidative stress was uncoupled eNOS. The presence of oxidative stress in CKD is well defined and is a contributing factor to the development of endothelial dysfunction. Treatments aimed at restoring vascular redox balance may be effective at reducing cardiovascular risk in CKD. In addition, supplementation with $\mathrm{BH}_{4}$ in humans with CKD may prevent eNOS uncoupling and reduce oxidative stress.

3.2. L-Arginine Deficiency. Nitric oxide synthesis relies on the amino acid substrate L-arginine. L-arginine synthesis primarily occurs in the proximal tubules of the renal cortex and is impaired with loss of functional renal mass [41]. Despite reduced production, the plasma concentration of L-arginine in patients with CKD appears to be maintained at normal levels [63]. The maintenance of plasma L-arginine levels in CKD may be a consequence of increased amino acid release into the blood due to skeletal muscle wasting [64], and impaired L-arginine transport due to increased uremic toxins $[65,66]$. Both mechanisms could potentially mask the decrease in L-arginine production by maintaining the plasma concentrations.

The intracellular concentration of L-arginine in endothelial cells is normally much higher than the Km of eNOS for L-arginine [67]. Despite the apparent cellular abundance of substrate, exogenous treatment with L-arginine has been effective at restoring endothelial function in other disease states including aging [68] and hypercholesterolemia [69, 70]. Cooke et al. [69] demonstrated that endothelialdependent relaxation of aortic rings was restored in hypercholesterolemic rabbits pretreated with an intravenous infusion of L-arginine. In 5/6 nephrectomized rats, both chronic and acute treatment with L-arginine improved blood pressure and restored endothelial-dependent relaxation of aortic rings [62]. This phenomenon, referred to as the "arginine paradox" $[67,71]$, was not observed, however, in patients receiving dialysis or in predialysis patients with severe renal failure [35]. In another study, infusion of L-arginine into the forearm improved the endothelial-dependent dilatory response to methacholine in both patients with CKD and aged-matched controls [30]. Age may have been a confounding factor in this study, however, since subjects were between 50 and 80 years of age.

L-arginine transport has been shown to be impaired by uremic toxins and may potentially explain why Larginine treatment has been ineffective in later stages of $\mathrm{CKD}$ (Figure 2). Endothelial cells cultured in uremic plasma had a reduced ability to transport L-arginine into the cells [65]. 


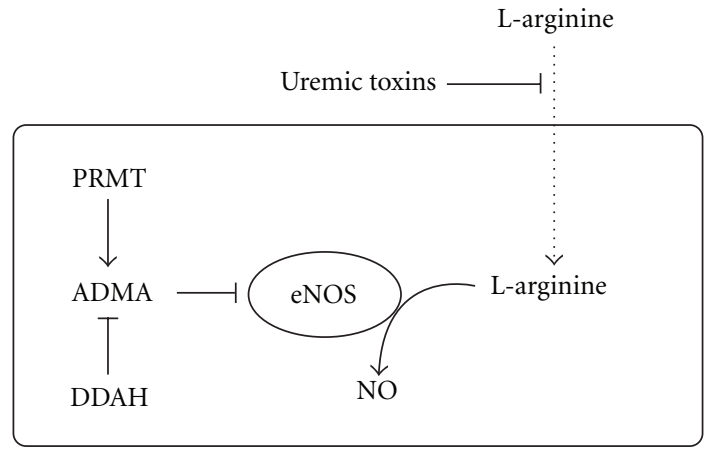

FIgUre 2: Potential mechanisms of L-arginine deficiency in chronic kidney disease (CKD). Asymmetric dimethylarginine (ADMA) is a competitive inhibitor of eNOS and ADMA levels are increased in CKD. Synthesis of ADMA occurs via protein arginine methyltransferase (PRMT) which may exhibit increased expression and activity in CKD. Expression of the enzyme dimethylarginine dimethylaminohydrolase (DDAH) may be decreased in CKD resulting in reduced degradation of ADMA. L-arginine transport into endothelial cells can be inhibited by increased levels of uremic toxins as disease progresses and result in reduced substrate availability for NO production.

When the same experiment was repeated with cells cultured in a synthetic solution containing uremic levels of urea, the same result was observed indicating that urea is an important inhibitor of L-arginine transport. The transport of L-arginine occurs through the cationic amino acid transporter CAT-1. Urea transport occurs through urea transporters (UT) that appear to be colocalized with CAT-1 in endothelial cells [66]. The movement of urea into the cell appears to be required in order to inhibit L-arginine transport. This mechanism may occur with the accumulation of uremic toxins as seen in CKD and lead to impaired Larginine transport and reduced substrate available for $\mathrm{NO}$ synthesis.

The uremic inhibition of L-arginine transport appears to occur in an "all-or-nothing" fashion $[41,65]$. Inhibition of L-arginine transport was only effective with a synthetic solution of urea at or above $15 \mathrm{mmol} / \mathrm{L}$ and not effective at lower concentrations [65]. Interestingly, when uremic plasma was diluted to achieve a lower urea concentration below this threshold, L-arginine transport was still inhibited. This suggests that other uremic toxins also contribute to transport inhibition and should be studied more extensively in order to elucidate their mechanisms.

Hyperhomocysteinemia reduced L-arginine uptake in cultured endothelial cells and impaired endothelialdependent relaxation in homocysteine-treated vessels and was associated with oxidative stress and increased nitrotyrosine levels [72]. Treatment with vitamin C restored cellular uptake of L-arginine while L-arginine treatment improved endothelial-dependent relaxation of aortic rings. CAT-1 expression was also attenuated in homocysteine-treated cells and was related to impaired NO production with no change in eNOS expression or activity.
Hyperhomocysteinemia is present in patients with CKD [4] and may contribute to the uremic inhibition of L-arginine transport in humans; however, this has not been studied.

The accumulation of uremic toxins in the plasma occurs progressively with loss of renal function. Increased levels of urea in humans has been correlated to decreased endothelialdependent dilation [31] and may be related to reduced Larginine transport. While L-arginine treatment has been ineffective at later stages of CKD and during hemodialysis, it is plausible that treatment in earlier stages, when the influence of uremic toxins is not as robust, may be effective at restoring endothelial function.

3.3. ADMA in CKD. Another potential contributor to endothelial dysfunction in CKD is the formation of the endogenous NOS inhibitors asymmetric dimethylarginine (ADMA) and $\mathrm{N}$-monomethylarginine (L-NMMA) $[73,74]$. ADMA and L-NMMA are the result of posttranscriptional methylation of L-arginine residues by protein arginine methyltransferases (PRMTs) and are released in their free form following protein hydrolysis. ADMA production is about 10-fold that of L-NMMA and is elevated in patients with chronic renal failure [75]. Plasma levels of ADMA predict progression to ESRD in patients with CKD [76] and ultimately predict adverse cardiovascular events in patients with mild to moderate CKD [77, 78]. ADMA is associated with impaired endothelial function in healthy adults [79], and has been shown to be inversely correlated to brachial artery FMD in patients with proteinuria and amyloidosis [26].

ADMA has been classified as a "uremic toxin" and exhibits adverse cardiovascular effects [4]. In healthy subjects infused with ADMA, heart rate and cardiac output were reduced while mean arterial pressure increased [80]. ADMA has also been linked to impaired endothelial function. Brachial artery FMD was impaired in healthy individuals treated with L-NMMA suggesting that a reduction in the ratio or L-arginine to methylated arginines competitively inhibits NO production in vivo [35]. The addition of L-arginine in these patients improved this balance and restored endothelial function. While L-arginine treatment is an effective strategy in restoring substrate balance and combating ADMA and L-NMMA in other disease models, its effectiveness in $\mathrm{CKD}$ is questionable due to the $\mathrm{L}$-arginine transport issues discussed above. It is therefore important to explore alternative strategies for reducing circulating levels of ADMA in kidney disease.

Interventions designed to lower ADMA production or increase ADMA clearance may be effective at improving endothelial function in CKD. Clearance of ADMA in the urine is impaired with renal damage and contributes to elevated plasma concentrations [81]; however, this is not the primary reason for elevated ADMA in CKD. Urinary clearance of ADMA did not explain elevations in plasma ADMA in an animal model of CKD [82]. Instead, increased PRMT activity and expression and reduced degradation of ADMA by dimethylarginine dimethylaminohydrolase (DDAH) are likely the major causes of increased ADMA in CKD [41] (Figure 2). PRMT expression and activity is 
increased in the presence of oxidized LDL cholesterol and results in increased production of ADMA in endothelial cells [83]. Antioxidant therapy with vitamin $\mathrm{E}$ has been shown to reduce ADMA levels in patients with CKD [84] and may be an effective treatment strategy to restore endothelial function. PRMT expression was increased in an animal model of CKD [82] and may be a potential therapeutic target to restore endothelial function.

DDAH converts ADMA to dimethylarginine and Lcitrulline and has been shown to decrease expression in an animal model of CKD [82]. A useful method for studying the role of ADMA in disease states is through the modification of DDAH enzyme expression in animal models. In mice infected with recombinant DDAH, NO synthesis was significantly increased while plasma ADMA concentrations were reduced compared to wild-type animals [85]. Endothelial-dependent relaxation was impaired in streptozotocin-induced diabetic rats and restored by overexpression DDAH [86]. When isolated mouse carotid artery sections were transfected with recombinant DDAH in the presence of exogenous ADMA, endothelial-dependent relaxation was improved compared to uninfected vessels [87]. ACE inhibition may also reduce ADMA levels and contribute to improved endothelial function. One study showed that 3 months of treatment with the ACE inhibitor Ramipril ( $5 \mathrm{mg} /$ day) or the angiotensin II receptor antagonist Valsartan $(160 \mathrm{mg} /$ day $)$ reduced ADMA levels and restored FMD in patients with nondiabetic CKD [26].

\section{Conclusion}

The endothelium represents the largest organ in the human body and is vital for the maintenance of vascular homeostasis. There is an urgent need to understand the mechanisms by which the endothelium becomes impaired in CKD in order to design more effective strategies for reducing cardiovascular risk. Chronic kidney disease is a worldwide health concern and leads to an accelerated risk of cardiovascular death. Because there are a significantly lower number of patients with later stages of CKD likely due to CVD-related death [3], earlier stages of CKD should be more extensively studied in order to better predict and prevent cardiovascular mortality in CKD. There is limited but convincing data supporting the role of endothelial dysfunction in earlier stages of CKD. Future research should focus on better understanding the mechanisms of endothelial dysfunction as well as development of effective interventions in earlier stages of CKD where the risk of cardiovascular death is most evident.

Evidence suggests that oxidative stress, L-arginine deficiency, and ADMA inhibition of NO synthesis all play roles in the pathogenesis of endothelial dysfunction in CKD. Based upon differences between patients with ESRD and earlier stages of CKD, it appears that there may be a point at which endothelial dysfunction becomes less reversible with advancing kidney disease [27]. Evidence from cell culture studies linking the impaired transport of L-arginine to uremic toxins supports this hypothesis and may be applied to other mechanisms of endothelial dysfunction. The increased uremic burden that accompanies late-stage CKD may trigger a "uremic switch" resulting in endothelial dysfunction that is less reversible due to an inability to transport L-arginine into endothelial cells. Once triggered, conventional therapies for treating endothelial dysfunction may become less effective leading to the increased prevalence of CVD seen in late-stage $\mathrm{CKD}$. It is therefore necessary to understand the pathogenesis of endothelial dysfunction in earlier stages of CKD in order to prevent the deterioration of vascular function.

\section{Acknowledgment}

This work was supported by National Institutes of Health Grants DK080469 and P20 RR016472-11.

\section{References}

[1] J. Coresh, B. C. Astor, T. Greene, G. Eknoyan, and A. S. Levey, "Prevalence of chronic kidney disease and decreased kidney function in the adult US population: Third National Health and Nutrition Examination Survey," American Journal of Kidney Diseases, vol. 41, no. 1, pp. 1-12, 2003.

[2] P. Muntner, J. He, L. Hamm, C. Loria, and P. K. Whelton, "Renal insufficiency and subsequent death resulting from cardiovascular disease in the United States," Journal of the American Society of Nephrology, vol. 13, no. 3, pp. 745-753, 2002.

[3] M. J. Sarnak, A. S. Levey, A. C. Schoolwerth et al., "Kidney disease as a risk factor for development of cardiovascular disease: a statement from the American Heart Association councils on kidney in cardiovascular disease, high blood pressure research, clinical cardiology, and epidemiology and prevention," Circulation, vol. 108, no. 17, pp. 2154-2169, 2003.

[4] R. Vanholder, R. De Smet, G. Glorieux et al., "Review on uremic toxins: classification, concentration, and interindividual variability," Kidney International, vol. 63, no. 5, pp. 1934-1943, 2003.

[5] A. S. Go, G. M. Chertow, D. Fan, C. E. McCulloch, and C. Y. Hsu, "Chronic kidney disease and the risks of death, cardiovascular events, and hospitalization," The New England Journal of Medicine, vol. 351, no. 13, pp. 1296-1370, 2004.

[6] N. M. Isbel, B. Haluska, D. W. Johnson, E. Beller, C. Hawley, and T. H. Marwick, "Increased targeting of cardiovascular risk factors in patients with chronic kidney disease does not improve atheroma burden or cardiovascular function," American Heart Journal, vol. 151, no. 3, pp. 745-753, 2006.

[7] J. Davignon and P. Ganz, "Role of endothelial dysfunction in atherosclerosis," Circulation, vol. 109, no. 23, pp. 27-32, 2004.

[8] S. Sitia, L. Tomasoni, F. Atzeni et al., "From endothelial dysfunction to atherosclerosis," Autoimmunity Reviews, vol. 9, no. 12 , pp. 830-834, 2010.

[9] D. Fischer, S. Rossa, U. Landmesser et al., "Endothelial dysfunction in patients with chronic heart failure is independently associated with increased incidence of hospitalization, cardiac transplantation, or death," European Heart Journal, vol. 26, no. 1, pp. 65-69, 2005.

[10] T. Heitzer, S. Baldus, Y. von Kodolitsch, V. Rudolph, and T. Meinertz, "Systemic endothelial dysfunction as an early predictor of adverse outcome in heart failure," Arteriosclerosis, Thrombosis, and Vascular Biology, vol. 25, no. 6, pp. 11741179, 2005.

[11] S. D. Katz, K. Hryniewicz, I. Hriljac et al., "Vascular endothelial dysfunction and mortality risk in patients with chronic heart failure," Circulation, vol. 111, no. 3, pp. 310-314, 2005. 
[12] M. Félétou and P. M. Vanhoutte, "Endothelial dysfunction: a multifaceted disorder," American Journal of Physiology, vol. 291, no. 3, pp. H985-H1002, 2006.

[13] F. Perticone, R. Maio, M. Perticone et al., "Endothelial dysfunction and subsequent decline in glomerular filtration rate in hypertensive patients," Circulation, vol. 122, no. 4, pp. 379-384, 2010.

[14] G. M. London, B. Pannier, M. Agharazii, A. P. Guerin, F. H. M. Verbeke, and S. J. Marchais, "Forearm reactive hyperemia and mortality in end-stage renal disease," Kidney International, vol. 65, no. 2, pp. 700-704, 2004.

[15] A. S. Levey, J. Coresh, K. Bolton et al., "K/DOQI clinical practice guidelines for chronic kidney disease: evaluation, classification, and stratification," American Journal of Kidney Diseases, vol. 39, supplement 1, no. 2, pp. S1-S266, 2002.

[16] M. Ketteler and P. H. Biggar, "Review article: getting the balance right: assessing causes and extent of vascular calcification in chronic kidney disease," Nephrology, vol. 14, no. 4, pp. 389 394, 2009.

[17] L. J. Ignarro, G. M. Buga, K. S. Wood, R. E. Byrns, and G. Chaudhuri, "Endothelium-derived relaxing factor produced and released from artery and vein is nitric oxide," Proceedings of the National Academy of Sciences of the United States of America, vol. 84, no. 24, pp. 9265-9269, 1987.

[18] R. M. J. Palmer, D. S. Ashton, and S. Moncada, "Vascular endothelial cells synthesize nitric oxide from L-arginine," Nature, vol. 333, no. 6174, pp. 664-666, 1988.

[19] P. J. Andrew and B. Mayer, "Enzymatic function of nitric oxide synthases," Cardiovascular Research, vol. 43, no. 3, pp. 521$531,1999$.

[20] S. Moncada and A. Higgs, "The L-arginine-nitric oxide pathway," The New England Journal of Medicine, vol. 329, no. 27, pp. 2002-2012, 1993.

[21] P. Kubes, M. Suzuki, and D. N. Granger, "Nitric oxide: an endogenous modulator of leukocyte adhesion," Proceedings of the National Academy of Sciences of the United States of America, vol. 88, no. 11, pp. 4651-4655, 1991.

[22] T. Scott-Burden and P. M. Vanhoutte, "The endothelium as a regulator of vascular smooth muscle proliferation," Circulation, vol. 87, no. 5, pp. V51-V55, 1993.

[23] S. Taddei, L. Ghiadoni, A. Virdis, D. Versari, and A. Salvetti, "Mechanisms of endothelial dysfunction: clinical significance and preventive non-pharmacological therapeutic strategies," Current Pharmaceutical Design, vol. 9, no. 29, pp. 2385-2402, 2003.

[24] D. S. Celermajer, K. E. Sorensen, V. M. Gooch et al., "Noninvasive detection of endothelial dysfunction in children and adults at risk of atherosclerosis," The Lancet, vol. 340, no. 8828, pp. 1111-1115, 1992.

[25] M. I. Yilmaz, J. Axelsson, A. Sonmez et al., "Effect of renin angiotensin system blockade on pentraxin 3 levels in type2 diabetic patients with proteinuria," Clinical Journal of the American Society of Nephrology, vol. 4, no. 3, pp. 535-541, 2009.

[26] M. I. Yilmaz, M. Saglam, A. Sonmez et al., "Improving proteinuria, endothelial functions and asymmetric dimethylarginine levels in chronic kidney disease: Ramipril versus valsartan," Blood Purification, vol. 25, no. 4, pp. 327-335, 2007.

[27] P. W. B. Nanayakkara, C. van Guldener, P. M. ter Wee et al., "Effect of a treatment strategy consisting of pravastatin, vitamin E, and homocysteine lowering on carotid intimamedia thickness, endothelial function, and renal function in patients with mild to moderate chronic kidney disease: results from the anti-oxidant therapy in chronic renal insufficiency
(ATIC) study," Archives of Internal Medicine, vol. 167, no. 12, pp. 1262-1270, 2007.

[28] M. Annuk, L. Lind, T. Linde, and B. Fellström, "Impaired endothelium-dependent vasodilatation in renal failure in humans," Nephrology Dialysis Transplantation, vol. 16, no. 2, pp. 302-306, 2001.

[29] M. Annuk, M. Zilmer, L. Lind, T. Linde, and B. Fellström, "Oxidative stress and endothelial function in chronic renal failure," Journal of the American Society of Nephrology, vol. 12, no. 12, pp. 2747-2752, 2001.

[30] M. Annuk, B. Fellström, and L. Lind, "Cyclooxygenase inhibition improves endothelium-dependent vasodilation in patients with chronic renal failure," Nephrology Dialysis Transplantation, vol. 17, no. 12, pp. 2159-2163, 2002.

[31] M. Annuk, I. Soveri, M. Zilmer, L. Lind, J. Hulthe, and B. Fellström, "Endothelial function, CRP and oxidative stress in chronic kidney disease," Journal of Nephrology, vol. 18, no. 6, pp. 721-726, 2005.

[32] L. Ghiadoni, A. Cupisti, Y. Huang et al., "Endothelial dysfunction and oxidative stress in chronic renal failure," Journal of Nephrology, vol. 17, no. 4, pp. 512-519, 2004.

[33] V. Costa-Hong, L. A. Bortolotto, V. Jorgetti, F. ConsolimColombo, E. M. Krieger, and J. J. Galvao de Lima, "Oxidative stress and endothelial dysfunction in chronic kidney disease," Arquivos Brasileiros de Cardiologia, vol. 92, no. 5, pp. 413-418, 2009.

[34] J. M. Cross, A. E. Donald, S. L. Nuttall, J. E. Deanfield, R. G. Woolfson, and R. J. MacAllister, "Vitamin C improves resistance but not conduit artery endothelial function in patients with chronic renal failure," Kidney International, vol. 63, no. 4, pp. 1433-1442, 2003.

[35] J. M. Cross, A. E. Donald, R. Kharbanda, J. E. Deanfield, R. G. Woolfson, and R. J. MacAllister, "Acute administration of L-arginine does not improve arterial endothelial function in chronic renal failure," Kidney International, vol. 60, no. 6, pp. 2318-2323, 2001.

[36] R. J. Schmidt, S. Yokota, T. S. Tracy, M. I. Sorkin, and C. Baylis, "Nitric oxide production is low in end-stage renal disease patients on peritoneal dialysis," American Journal of Physiology, vol. 276, no. 5, pp. F794-F797, 1999.

[37] S. T. W. Morris, J. J. V. McMurray, A. Spiers, and A. G. Jardine, "Impaired endothelial function in isolated human uremic resistance arteries," Kidney International, vol. 60, no. 3, pp. 1077-1082, 2001.

[38] J. A. Kari, A. E. Donald, D. T. Vallance et al., "Physiology and biochemistry of endothelial function in children with chronic renal failure," Kidney International, vol. 52, no. 2, pp. 468-472, 1997.

[39] G. Hussein, Y. Bughdady, M. E. Kandil, H. M. Bazaraa, and H. Taher, "Doppler assessment of brachial artery flow as a measure of endothelial dysfunction in pediatric chronic renal failure," Pediatric Nephrology, vol. 23, no. 11, pp. 2025-2030, 2008.

[40] A. C. Wilson, E. Urbina, S. A. Witt, B. J. Glascock, T. R. Kimball, and M. Mitsnefes, "Flow-mediated vasodilatation of the brachial artery in children with chronic kidney disease," Pediatric Nephrology, vol. 23, no. 8, pp. 1297-1302, 2008.

[41] C. Baylis, "Nitric oxide deficiency in chronic kidney disease," American Journal of Physiology, vol. 294, no. 1, pp. F1-F9, 2008.

[42] Y. Higashi, K. Noma, M. Yoshizumi, and Y. Kihara, "Endothelial function and oxidative stress in cardiovascular diseases," Circulation Journal, vol. 73, no. 3, pp. 411-418, 2009. 
[43] T. J. Guzik and D. G. Harrison, "Vascular NADPH oxidases as drug targets for novel antioxidant strategies," Drug Discovery Today, vol. 11, no. 11-12, pp. 524-533, 2006.

[44] B. P. Oberg, E. McMenamin, F. L. Lucas et al., "Increased prevalence of oxidant stress and inflammation in patients with moderate to severe chronic kidney disease," Kidney International, vol. 65, no. 3, pp. 1009-1016, 2004.

[45] M. I. Yilmaz, M. Saglam, K. Caglar et al., "The determinants of endothelial dysfunction in CKD: oxidative stress and asymmetric dimethylarginine," American Journal of Kidney Diseases, vol. 47, no. 1, pp. 42-50, 2006.

[46] C. K. Chen, J. M. Liaw, J. G. Juang, and T. E. H. Lin, "Antioxidant enzymes and trace elements in hemodialyzed patients," Biological Trace Element Research, vol. 58, no. 1-2, pp. 149-157, 1997.

[47] N. Takahashi, S. Morimoto, M. Okigaki et al., "Decreased plasma level of vitamin C in chronic kidney disease: comparison between diabetic and non-diabetic patients," Nephrology, Dialysis, Transplantation, vol. 26, no. 4, pp. 1252-1257, 2011.

[48] E. Linden, W. Cai, J. C. He et al., "Endothelial dysfunction in patients with chronic kidney disease results from advanced glycation end products (AGE)-mediated inhibition of endothelial nitric oxide synthase through RAGE activation," Clinical Journal of the American Society of Nephrology, vol. 3, no. 3, pp. 691-698, 2008.

[49] S. Vasdev, V. Gill, and P. Singal, "Role of advanced glycation end products in hypertension and atherosclerosis: therapeutic implications," Cell Biochemistry and Biophysics, vol. 49, no. 1, pp. 48-63, 2007.

[50] G. Hasdan, S. Benchetrit, G. Rashid, J. Green, J. Bernheim, and M. Rathaus, "Endothelial dysfunction and hypertension in 5/6 nephrectomized rats are mediated by vascular superoxide," Kidney International, vol. 61, no. 2, pp. 586-590, 2002.

[51] M. Tepel, M. van der Giet, M. Statz, J. Jankowski, and W. Zidek, "The antioxidant acetylcysteine reduces cardiovascular events in patients with end-stage renal failure: a randomized, controlled trial," Circulation, vol. 107, no. 7, pp. 992-995, 2003.

[52] M. Boaz, S. Smetana, T. Weinstein et al., "Secondary prevention with antioxidants of cardiovascular disease in endstage renal disease (SPACE): randomised placebo-controlled trial," The Lancet, vol. 356, no. 9237, pp. 1213-1218, 2000.

[53] N. F. Wiernsperger, "Oxidative stress as a therapeutic target in diabetes: revisiting the controversy," Diabetes and Metabolism, vol. 29, no. 6, pp. 579-585, 2003.

[54] S. Shelkovnikov, S. M. Summers, R. Elahimehr, G. Adams, R. E. Purdy, and N. D. Vaziri, "Effect of exercise training on aortic tone in chronic renal insufficiency," American Journal of Hypertension, vol. 21, no. 5, pp. 564-569, 2008.

[55] D. R. Seals, C. A. DeSouza, A. J. Donato, and H. Tanaka, "Habitual exercise and arterial aging," Journal of Applied Physiology, vol. 105, no. 4, pp. 1323-1332, 2008.

[56] N. D. Vaziri, Z. Ni, F. Oveisi, K. Liang, and R. Pandian, "Enhanced nitric oxide inactivation and protein nitration by reactive oxygen species in renal insufficiency," Hypertension, vol. 39, no. 1, pp. 135-141, 2002.

[57] G. A. Kaysen and J. P. Eiserich, "The role of oxidative stressaltered lipoprotein structure and function and microinflammation on cardiovascular risk in patients with minor renal dysfunction," Journal of the American Society of Nephrology, vol. 15, no. 3, pp. 538-548, 2004.
[58] M. Goicoechea, S. Garcia de Vinuesa, U. Verdalles et al., "Effect of allopurinol in chronic kidney disease progression and cardiovascular risk," Clinical Journal of the American Society of Nephrology, vol. 5, no. 8, pp. 1388-1393, 2010.

[59] J. Vśquez-Vivar, B. Kalyanaraman, and P. Martásek, “The role of tetrahydrobiopterin in superoxide generation from eNOS: enzymology and physiological implications," Free Radical Research, vol. 37, no. 2, pp. 121-127, 2003.

[60] A. C. F. Gorren and B. Mayer, "Tetrahydrobiopterin in nitric oxide synthesis: a novel biological role for pteridines," Current Drug Metabolism, vol. 3, no. 2, pp. 133-157, 2002.

[61] E. Podjarny, G. Hasdan, J. Bernheim et al., "Effect of chronic tetrahydrobiopterin supplementation on blood pressure and proteinuria in 5/6 nephrectomized rats," Nephrology Dialysis Transplantation, vol. 19, no. 9, pp. 2223-2227, 2004.

[62] K. Yamamizu, K. Shinozaki, K. Ayajiki, M. Gemba, and T. Okamura, "Oral administration of both tetrahydrobiopterin and L-arginine prevents endothelial dysfunction in rats with chronic renal failure," Journal of Cardiovascular Pharmacology, vol. 49, no. 3, pp. 131-139, 2007.

[63] A. A. Reyes, I. E. Karl, and S. Klahr, "Role of arginine in health and in renal disease," American Journal of Physiology, vol. 267, no. 3, pp. F331-F346, 1994.

[64] H. Moradi, V. Kwok, and N. D. Vaziri, "Effect of chronic renal failure on arginase and argininosuccinate synthetase expression," American Journal of Nephrology, vol. 26, no. 3, pp. 310-318, 2006.

[65] S. Xiao, L. Wagner, J. Mahaney, and C. Baylis, "Uremic levels of urea inhibit L-arginine transport in cultured endothelial cells," American Journal of Physiology, vol. 280, no. 6, pp. F989-F995, 2001.

[66] L. Wagner, J. D. Klein, J. M. Sands, and C. Baylis, "Urea transporters are distributed in endothelial cells and mediate inhibition of L-arginine transport," American Journal of Physiology, vol. 283, no. 3, pp. F578-F582, 2002.

[67] T. A. Hardy and J. M. May, "Coordinate regulation of Larginine uptake and nitric oxide synthase activity in cultured endothelial cells," Free Radical Biology and Medicine, vol. 32, no. 2, pp. 122-131, 2002.

[68] S. M. Bode-Böger, J. Muke, A. Surdacki, G. Brabant, R. H. Böger, and J. C. Frölich, "Oral L-arginine improves endothelial function in healthy individuals older than 70 years," Vascular Medicine, vol. 8, no. 2, pp. 77-81, 2003.

[69] J. P. Cooke, N. A. Andon, X. J. Girerd, A. T. Hirsch, and M. A. Creager, "Arginine restores cholinergic relaxation of hypercholesterolemic rabbit thoracic aorta," Circulation, vol. 83, no. 3, pp. 1057-1062, 1991.

[70] P. Clarkson, M. R. Adams, A. J. Powe et al., "Oral L-arginine improves endothelium-dependent dilation in hypercholesterolemic young adults," Journal of Clinical Investigation, vol. 97, no. 8, pp. 1989-1994, 1996.

[71] R. H. Böger, "Asymmetric dimethylarginine, an endogenous inhibitor of nitric oxide synthase, explains the "L-arginine paradox" and acts as a novel cardiovascular risk factor," Journal of Nutrition, vol. 134, no. 10, pp. 2842S-2847S, 2004.

[72] L. Jin, R. B. Caldwell, T. Li-Masters, and R. W. Caldwell, "Homocysteine induces endothelial dysfunction via inhibition of arginine transport," Journal of Physiology and Pharmacology, vol. 58, no. 2, pp. 191-206, 2007.

[73] J. T. Kielstein and C. Zoccali, "Asymmetric dimethylarginine: a cardiovascular risk factor and a uremic toxin coming of age?" American Journal of Kidney Diseases, vol. 46, no. 2, pp. 186202, 2005. 
[74] C. Baylis, "Arginine, arginine analogs and nitric oxide production in chronic kidney disease," Nature Clinical Practice Nephrology, vol. 2, no. 4, pp. 209-220, 2006.

[75] P. Vallance, A. Leone, A. Calver, J. Collier, and S. Moncada, "Accumulation of an endogenous inhibitor of nitric oxide synthesis in chronic renal failure," The Lancet, vol. 339, no. 8793, pp. 572-575, 1992.

[76] D. Fliser, F. Kronenberg, J. T. Kielstein et al., "Asymmetric dimethylarginine and progression of chronic kidney disease: the mild to moderate kidney disease study," Journal of the American Society of Nephrology, vol. 16, no. 8, pp. 2456-2461, 2005.

[77] P. Ravani, G. Tripepi, F. Malberti, S. Testa, F. Mallamaci, and C. Zoccali, "Asymmetrical dimethylarginine predicts progression to dialysis and death in patients with chronic kidney disease: a competing risks modeling approach," Journal of the American Society of Nephrology, vol. 16, no. 8, pp. 2449-2455, 2005.

[78] J. M. Young, N. Terrin, X. Wang et al., "Asymmetric dimethylarginine and mortality in stages 3 to 4 chronic kidney disease," Clinical Journal of the American Society of Nephrology, vol. 4, no. 6, pp. 1115-1120, 2009.

[79] M. Juonala, J. S. A. Viikari, G. Alfthan et al., "Brachial artery flow-mediated dilation and asymmetrical dimethylarginine in the cardiovascular risk in young Finns study," Circulation, vol. 116, no. 12, pp. 1367-1373, 2007.

[80] V. Achan, M. Broadhead, M. Malaki et al., "Asymmetric dimethylarginine causes hypertension and cardiac dysfunction in humans and is actively metabolized by dimethylarginine dimethylaminohydrolase," Arteriosclerosis, Thrombosis, and Vascular Biology, vol. 23, no. 8, pp. 1455-1459, 2003.

[81] J. T. Kielstein, R. H. Böger, S. M. Bode-Böger et al., "Asymmetric dimethylarginine plasma concentrations differ in patients with end-stage renal disease: relationship to treatment method and atherosclerotic disease," Journal of the American Society of Nephrology, vol. 10, no. 3, pp. 594-600, 1999.

[82] K. Matsuguma, S. Ueda, S. I. Yamagishi et al., "Molecular mechanism for elevation of asymmetric dimethylarginine and its role for hypertension in chronic kidney disease," Journal of the American Society of Nephrology, vol. 17, no. 8, pp. 21762183, 2006.

[83] R. H. Böger, K. Sydow, J. Borlak et al., "LDL cholesterol upregulates synthesis of asymmetrical dimethylarginine in human endothelial cells: involvement of S-adenosylmethioninedependent methyltransferases," Circulation Research, vol. 87, no. 2, pp. 99-105, 2000.

[84] R. Saran, J. E. Novak, A. Desai et al., "Impact of vitamin E on plasma asymmetric dimethylarginine (ADMA) in chronic kidney disease (CKD): a pilot study," Nephrology Dialysis Transplantation, vol. 18, no. 11, pp. 2415-2420, 2003.

[85] H. Dayoub, V. Achan, S. Adimoolam et al., "Dimethylarginine dimethylaminohydrolase regulates nitric oxide synthesis: genetic and physiological evidence," Circulation, vol. 108, no. 24, pp. 3042-3047, 2003.

[86] C. W. Lu, Z. Guo, M. Feng, Z. Z. Wu, Z. M. He, and Y. Xiong, "Ex vivo gene transferring of human dimethylarginine dimethylaminohydrolase-2 improved endothelial dysfunction in diabetic rat aortas and high glucose-treated endothelial cells," Atherosclerosis, vol. 209, no. 1, pp. 66-73, 2010.

[87] B. Torondel, M. Nandi, P. Kelly, B. Wojciak-Stothard, I. Fleming, and J. Leiper, "Adenoviral-mediated overexpression of DDAH improves vascular tone regulation," Vascular Medicine, vol. 15 , no. 3, pp. 205-213, 2010. 


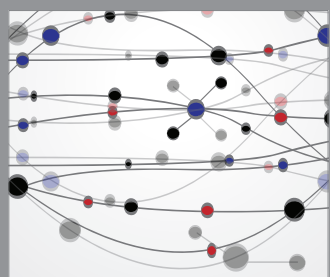

The Scientific World Journal
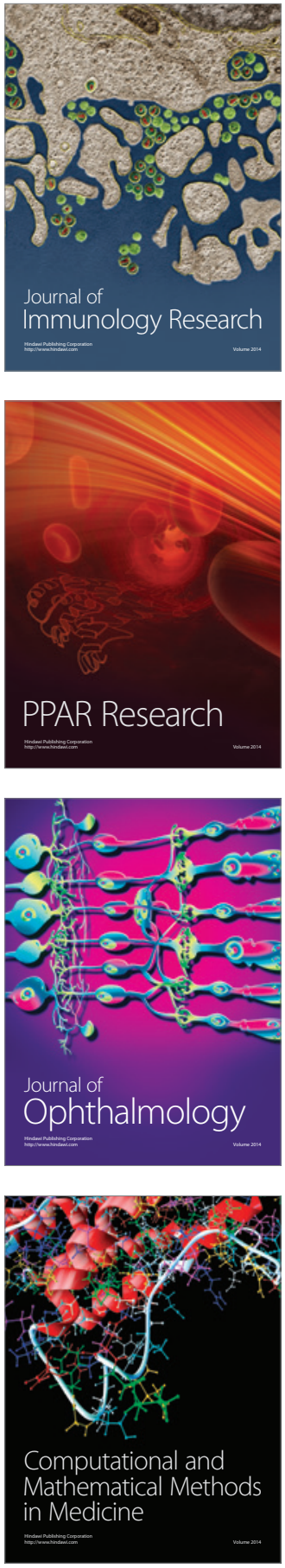

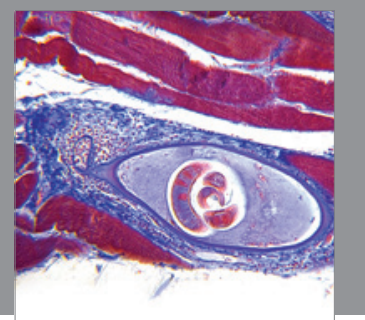

Gastroenterology

Research and Practice
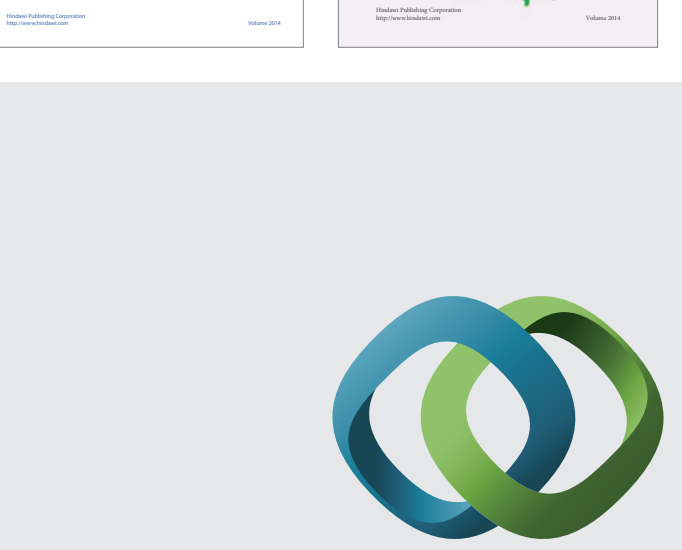

\section{Hindawi}

Submit your manuscripts at

http://www.hindawi.com
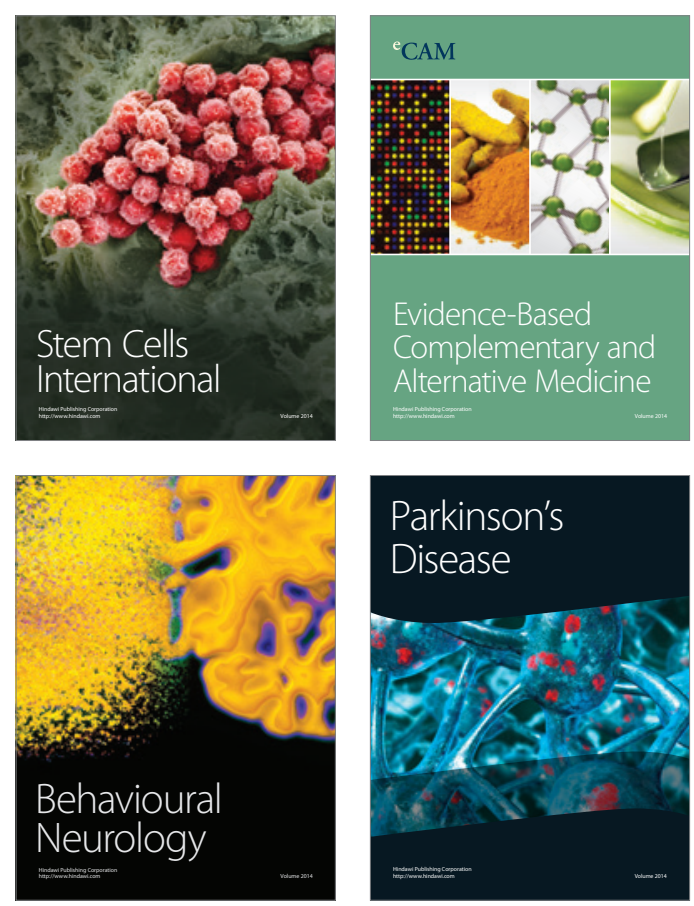

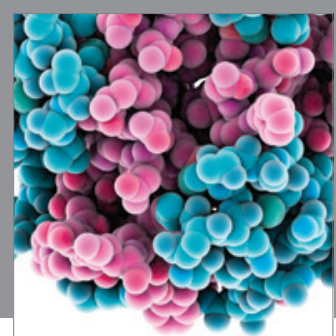

Journal of
Diabetes Research

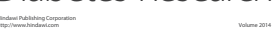

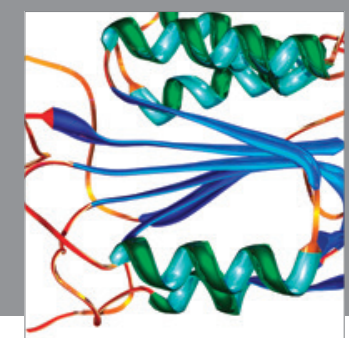

Disease Markers
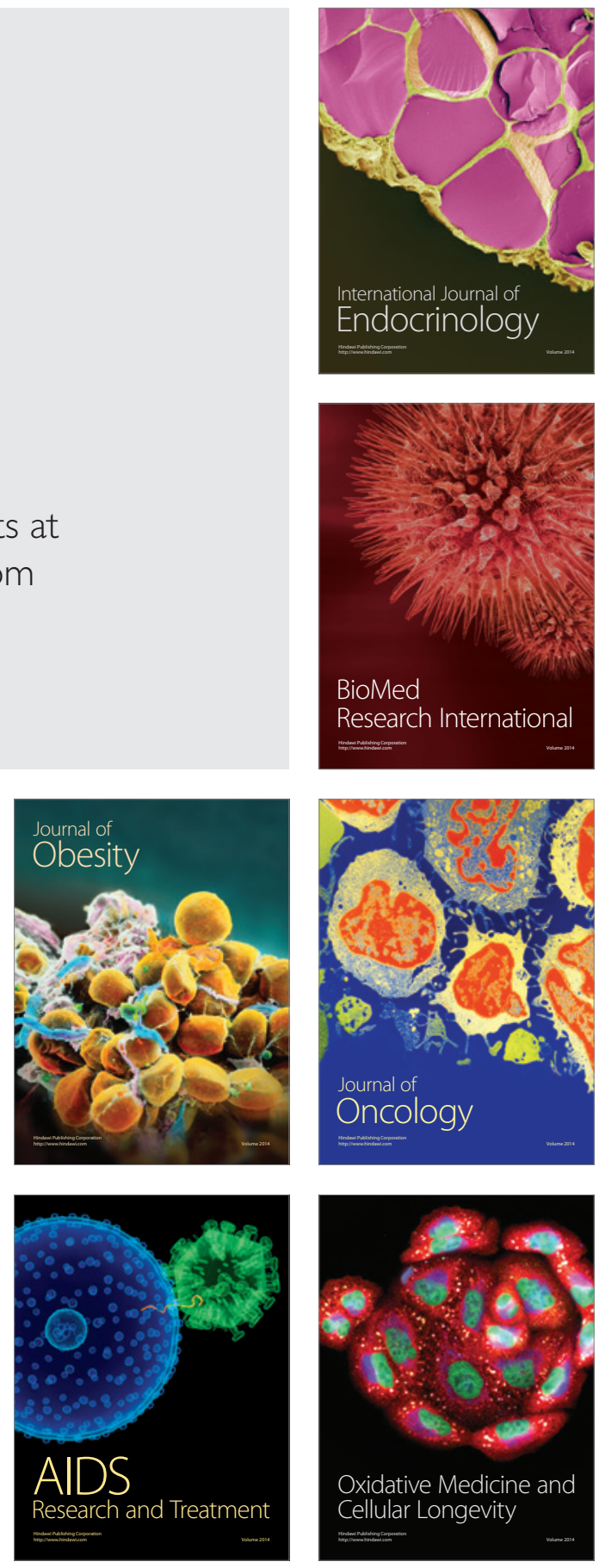